\section{Diagnosis of Clostridium difficile infection: comparison of four methods on specimens collected in Cary-Blair trans- port medium and tcdB PCR on fresh versus frozen samples}

\author{
Noah A. Brown, William D. LeBar, \\ Carol L. Young, Rosemary E. Hankerd, \\ Duane W. Newton
}

Department of Pathology, University of Michigan Health System, Ann Arbor, MI, USA

\section{Abstract}

Clostridium difficile infection (CDI) caused by toxigenic strains of $C$. difficile is primarily a nosocomial infection with increasing prevalence. Stool specimens are typically collected in Cary-Blair transport medium to maximize culture-based detection of common stool pathogens. The goal of this study was to establish an analytically accurate and efficient algorithm for the detection of CDI in our patient population using samples collected in Cary-Blair transport medium. In addition, we wished to determine whether the sensitivity and specificity of PCR was affected by freezing samples before testing. Using 357 specimens, we compared four methods: enzyme immunoassay for the antigen glutamate dehydrogenase (Wampole $^{\mathrm{TM}}$ C. DIFF CHEK-60 Assay, GDH), toxin A and B enzyme immunoassay (Remel ProSpecT'T $C$. difficile Toxin A/B Microplate Assay, Toxin EIA), cell culture cytotoxicity neutralization assay (Bartels ${ }^{\mathrm{TM}}$ Cytotoxicity Assay, $\mathrm{CT}$ ), and real-time PCR targeting the toxin B gene (BD GeneOhm ${ }^{\mathrm{TM}}$ Cdiff Assay, PCR). The analytic sensitivity and specificity of each as determined using a combined gold standard were as follows: GDH, $100 \%$ and $93.2 \%$; Toxin EIA, $82.9 \%$ and $82.9 \%$; CT, $100 \%$ and $100 \%$; PCR (performed on frozen specimens) $74.3 \%$ and $96.6 \%$; respectively. However, the sensitivity and specificity of PCR improved to $100 \%$ when performed on 50 fresh stool samples collected in Cary-Blair. While CT remains a sensitive method for the detection of CDI, GDH offers an excellent initial screening method to rule out CDI. While the performance of each assay did not appear to be affected by collection in Cary-Blair medium, PCR performed better using fresh specimens.

\section{Introduction}

Clostridium difficile is an anaerobic, spore- forming, gram-positive bacterium which causes a wide range of infections including severe diarrhea, pseudomembranous colitis, and toxic megacolon. $C$. difficile infection (CDI) often follows the eradication of normal colonic flora and is a common nosocomial infection. From 1993 to 2003 the incidence of CDI among hospitalized patients doubled ${ }^{1}$ and is estimated to cost $\$ 3,669$ to $\$ 7,234$ per patient hospitalization. ${ }^{2-4} C$. difficile is capable of producing an enterotoxin (toxin A), a cytotoxin (toxin B), and an actin-specific ADP-ribosyltransferase (binary toxin). ${ }^{5}$ The most common methods currently employed within clinical laboratories to diagnosis CDI are enzyme immunoassays (EIA) designed to detect either toxin $\mathrm{A}$ alone or toxins $\mathrm{A}$ and $\mathrm{B}$ together. Although toxin EIA is a low cost option with a rapid turn-around time, this method is unreliable, with sensitivities ranging from $38-98 \% .^{5-15}$

A testing modality with a high negative predictive value is needed to rule out $C$. difficile infection among the many patients with diarrhea from other causes. An assay with a high positive predictive value is also needed to avoid the unwarranted prescription of antibiotics and patients unnecessarily subjected to isolation precautions. As early treatment improves the potential success of therapy, and immediate isolation is necessary to prevent the spread of the infection, a rapid turnaround time is also a priority for any testing choice. Finally, the increasingly high volume of testing performed demands testing with a low unit cost and minimal workforce time.

The need for a testing methodology with superior performance characteristics has resulted in the development of new assays, as well as the resurgence of older assays, for the detection of toxin-producing $C$. difficile. Alternative assays include enzyme immunoassays for the detection of glutamate dehydrogenase (GDH) antigen expressed on the surface of $C$. difficile, cell culture cytotoxicity neutralization assay, toxigenic culture, and real-time PCR amplification of the toxin B gene.

Cary-Blair medium is a non-nutritive medium for stool specimens that prevents overgrowth of Enterobacteriaceae and preserves common stool pathogens including Salmonella, Shigella, Vibrio and Yersinia enterocolitica. While GDH antigen EIA and toxin EIA are well-studied on this medium, both cytotoxicity neutralization assays and PCR are recommended for fresh samples without preservative. The purpose of this study was to establish an analytically accurate and efficient algorithm for the detection of CDI in our patient population when performed on specimens collected in Cary-Blair medium. In addition, we sought to determine whether the sensitivity and specificity of PCR was affected by freezing samples before testing.
7Correspondence: Duane W. Newton, Department of Pathology, University of Michigan Health System, 1500 E. Medical Center Drive, UH2G332, Ann Arbor, Michigan 48109, USA

Tel. +734.936.6847 - Fax: 734.647.9093

E-mail: dnewton@med.umich.edu

Key words: clostridium difficile, cary-Blair, frozen, PCR, glutamate dehydrogenase.

Acknowledgements: Support in the form of materials for PCR provided by BD GeneOhm ${ }^{\mathrm{TM}}$ and materials for GDH antigen testing provided by Inverness Medical.

Contributions: NAB, manuscript composition and revision, data conception and design, data analysis and interpretation, manuscript final approval; WDL, CLY, REH, REH, DWN, manuscript conception and design, data acquisition, analysis and interpretation, manuscript revision.

Conflict of interest: the authors report no conflicts of interest.

Received for publication: 4 February 2011.

Revision received: 15 April 2011.

Accepted for publication: 20 April 2011.

This work is licensed under a Creative Commons Attribution 3.0 License (by-nc 3.0).

(C) Copyright N.A. Brown et al., 2011

Licensee PAGEPress, Italy

Infectious Disease Reports 2011; 3:e5

doi:10.4081/idr.2011.e5

\section{Materials and Methods}

A total of 357 stool specimens collected in Cary-Blair transport (Para-Pak ${ }^{\mathrm{TM}} \mathrm{C} \& \mathrm{~S}$ transport media; Meridian Diagnostics, Cincinnati, $\mathrm{OH})$. There were no specific criteria pertaining to the character of stool specimens as all specimens were received in liquid transport medium. Specimens were tested by four different methods: Glutamate dehydrogenase antigen EIA (Wampole $^{\mathrm{TM}}$ C. DIFF CHEK-60 Assay; Iverness Medical, Princeton, NJ), $C$. difficile toxin $\mathrm{A} / \mathrm{B}$ EIA (Remel ProSpecT ${ }^{\mathrm{TM}} C$. difficile Toxin A/B Microplate Assay; Lenexa, KS), cell culture cytotoxicity neutralization (Bartels ${ }^{\mathrm{TM}}$ Cytotoxicity Assay; Carlsbad, CA), and real-time PCR for toxin B $(t c d B)$ gene (BD GeneOhm ${ }^{\mathrm{TM}}$ Cdiff Assay; Franklin Lakes, New Jersey). GDH antigen EIA, toxin EIA, and cell culture cytotoxicity testing was performed upon receipt of specimens. For practical reasons, residual stool specimens in Cary-Blair were frozen at $-80^{\circ} \mathrm{C}$ for testing by PCR at a later time. In order to determine the impact of freezing on PCR results, an additional 50 fresh specimens collected in CaryBlair were tested upon arrival in the lab with GDH antigen EIA, toxin EIA, and PCR. 
All assays were performed according to the manufacturers' instructions with the exception of specimens being collected in Cary-Blair transport medium (rather than raw), and freezing samples for PCR. Frozen specimens were allowed to thaw completely at room temperature. Before starting the assay, the thawed transport tubes were vortex-mixed at high speed for 15 seconds and then a sterile dry swab was dipped into the transport material. Excess stool was removed and the swab was placed in a sample buffer tube. PCR for the $t c d B$ gene was then performed according to the manufacturer's instructions.

Results from the Remel ProSpecT ${ }^{\mathrm{TM}} C$. difficile Toxin A/B Microplate Assay were read spectrophotometrically and interpreted as described in the package insert. Briefly, the absorbance of each well was determined by scanning the microplate with spectrophotometer to read bichromatically at $450 / 620-650 \mathrm{~nm}$. All results with an absorbance below 0.08 were reported negative and all results with an absorbance above 0.149 were reported positive. An absorbance between 0.08 and 0.149 was considered equivocal and resulted in repeat testing of the sample in duplicate. Samples with two out of three results above 0.08 were reported positive, while samples with two out of three results below 0.08 were reported negative.

Each of the four methods was evaluated based on their ability to detect toxin-producing $C$. difficile. Any sample testing positive by all 4 methods was deemed positive. Likewise, any sample testing negative by all 4 was deemed negative. Samples that were positive by GDH but negative by all other methods were interpreted to reflect a sample with non-toxin producing $C$. difficile and were therefore considered negative. Samples positive by three different methods (negative by only one) were deemed positive. Based on the high specificity of cell culture cytotoxicity neutralization in previous studies, all samples positive by this method were deemed true positives. All other samples were deemed negative for purposes of calculating performance characteristics. For the 50 fresh samples tested by GDH, toxin EIA and PCR, specimens were considered positive if the GDH and either the toxin EIA or PCR were concurrently positive.

\section{Results}

Of the 357 samples subjected to $C$. difficile testing, 35 (9.8\%) satisfied the criteria to be considered true positives. A breakdown of the complete results is shown in Table 1. Analytic sensitivities, specificities, positive and negative predictive values of all four methods are shown in Table 2.

\section{GDH antigen testing}

Fifty-seven specimens (16.0\%) were positive by GDH antigen enzyme immunoassay. There were no samples that were positive for toxin-producing $C$. difficile but negative by GDH testing, resulting in a sensitivity and negative predictive value of $100 \%$. Twelve samples (3.4\%) were positive by GDH and negative by the other three methods, consistent with nontoxin producing $C$. difficile. Eight samples (2.2\%) were positive by GDH and PCR only and were deemed negative for purposes of calculating performance characteristics. The specificity was $93.2 \%$ and the positive predictive value was $61.4 \%$.

\section{Toxin A/B EIA}

Sixty-nine specimens (19.3\%) were positive by toxin enzyme immunoassay. Three samples (0.8\%) tested negative via toxin EIA but positive by all three other methods. Three additional samples $(0.8 \%)$ were positive by GDH testing and cell culture cytotoxicity neutralization. Based on these false negative results, the sensitivity and negative predictive value of toxin EIA were $82.9 \%$ and $97.9 \%$. Thirty-eight samples $(10.6 \%)$ were positive by toxin EIA but negative by all three other methods. The specificity and positive predictive value were $87.6 \%$ and $42.0 \%$ respectively.

\section{Cell culture cytotoxicity neutralization}

Thirty-five samples $(9.8 \%)$ were positive by cell culture cytotoxicity neutralization assay.
There were no samples negative via CT but positive by the other three methods. Therefore, the sensitivity and negative predictive value were each $100 \%$. Because there were also no CT false positives, the specificity and positive predictive values were both $100 \%$.

\section{Toxin B PCR}

Thirty-eight samples (10.6\%) were positive by polymerase chain reaction for the toxin $\mathrm{B}$ gene. Six samples (1.7\%) were PCR negative but positive by the other three methods (interpreted as a PCR false negative). Three additional samples $(0.8 \%)$ were PCR negative, but positive by GDH testing and cell culture cytotoxicity neutralization. The resulting sensitivity of PCR was $74.3 \%$ with a negative predictive value of $97.2 \%$. One sample was positive by PCR but negative by all three other methods (deemed a false positive). Eight samples (2.2\%) were positive by GDH and PCR only and were deemed negative by our criteria. Two samples were positive by Toxin A/B EIA and PCR only and were also deemed negative. The specificity and positive predictive value were determined to be $96.6 \%$ and $70.3 \%$ respectively.

\section{PCR on fresh specimens}

A total of 50 fresh stool samples in Cary Blair were tested by the GDH, toxin EIA, and PCR. Of these samples, 12 were classified as true positives based on the study criteria and all were detected by the PCR assay. An additional 2 samples were only positive by the PCR assay only.

Table 1. Aggregate results of stool samples evaluated by each $C$. difficile testing modality.

\begin{tabular}{|c|c|c|c|c|c|}
\hline$\nabla$ & & Assay & & & \\
\hline & GDH & Toxin EIA & $\mathrm{CT}$ & PCR & Observations ${ }^{b}$ \\
\hline True positives & + & + & + & + & $23(6.4 \%)$ \\
\hline & + & - & + & + & $3(0.8 \%)$ \\
\hline & + & + & + & - & $6(1.7 \%)$ \\
\hline & + & - & + & - & $3(0.8 \%)$ \\
\hline False positives & - & + & - & + & $2(0.6 \%)$ \\
\hline & + & - & - & + & $8(2.2 \%)$ \\
\hline & + & - & - & - & $12(3.4 \%)$ \\
\hline & - & + & - & - & $38(10.6 \%)$ \\
\hline & - & - & - & + & $1(0.3 \%)$ \\
\hline True negatives & - & - & - & - & $261(73.1 \%)$ \\
\hline
\end{tabular}

aGDH: glutamate dehydrogenase antigen assay; Toxin EIA, C. difficile toxin A/B enzyme immunoassay; CT, cell-culture cytotoxicity; PCR, toxin B gene detection by PCR; 'Number of samples (\% of total tested) with the indicated results.

Table 2. Performance characteristics of different $C$. difficile testing modalities compared to a combined standard. ${ }^{\mathrm{a}}$

\begin{tabular}{|c|c|c|c|c|}
\hline \multirow[b]{2}{*}{ Assay $^{b}$} & \multicolumn{4}{|c|}{ Performance parameter, \% (95\% confidence interval) } \\
\hline & Sensitivity & Specificity & PPVc & NPV d \\
\hline GDH & $100(87.7-100)$ & $93.2(89.7-95.6)$ & $61.4(47.6-73.7)$ & $100(98.4-100)$ \\
\hline Toxin EIA & $82.9(65.7-92.8)$ & $87.6(83.4-90.9)$ & $42.0(30.4-54.5)$ & $97.9(95.3-99.2)$ \\
\hline CT & $100(87.7-100)$ & $100(98.5-100)$ & $100(87.7-100)$ & $100(98.5-100)$ \\
\hline PCR & $74.3(56.4-86.9)$ & $96.6(93.8-98.2)$ & $70.3(52.8-83.6)$ & $97.2(94.5-98.6)$ \\
\hline
\end{tabular}

aSee Methods for complete description of standard; ${ }^{\mathrm{b}} \mathrm{GDH}$ : glutamate dehydrogenase antigen assay; ${ }^{\circ}$ Positive predictive value; ${ }^{\mathrm{N}}$ Negative predictive value. toxin EIA, C. difficile toxin A/B enzyme immunoassay; CT, cell-culture cytotoxicity; PCR, toxin B gene detection by PCR. 


\section{Retrospective algorithm}

A retrospective analysis of five different algorithms using the data generated in this evaluation is depicted in Table 3. Algorithms include 3 two-step algorithms (GDH followed by either toxin EIA, CT, or PCR for GDH-positive results), and 2 three-step algorithms (GDH followed by toxin EIA of GDH-positive results followed by either CT or PCR of toxin EIA-negative results). Although algorithms which included CT performed the best $(100 \%$ for all performance parameters), a three-step algorithm which included GDH, EIA and PCR exhibited a high sensitivity (91.4\%) and an excellent NPV (99.1\%).

\section{Discussion}

The goal of this study was to determine whether a method or combination of methods could accurately and efficiently diagnose CDI when performed on stool specimens submitted Cary-Blair transport medium. This study demonstrates that GDH represents a sensitive screening method and that CT is a sensitive and specific means of confirming GDH-positive results. Alternatively, PCR is a sensitive and specific method when performed on fresh specimens. The collection of stool specimens in Cary-Blair medium did not impact the accuracy of either CT or PCR.

The most common method currently employed within clinical laboratories to diagnosis CDI is the toxin EIA. This method is a low cost option with a rapid turn-around time. However, in this study, the sensitivity and specificity of toxin EIA was $82.9 \%$ and $87.6 \%$, respectively. As a result of the relatively low prevalence of disease (9.8\%), and a substantial number of specimens that were positive only by EIA, the positive predictive value was very poor $(42.0 \%)$. A patient with a positive toxin EIA would likely be subjected to antibiotic treatment and isolation precautions even though most positive results represented false positives. These false positive results may result from antibody cross-reactivity with antigens other than the $C$. difficile toxin or, less likely, from cross-contamination during the washing steps. While a number of different toxin EIA assays are available, each with different monoclonal antibodies, the results of this study are in keeping with results from other studies. ${ }^{5-15}$

Another EIA technique detects GDH antigen on the surface of the $C$. difficile. Several studies have shown the GDH antigen EIA to be substantially more sensitive than toxin EIA. , $, 712,16,17$ The sensitivity and negative predictive value of GDH in this study were each $100 \%$. In our patient population, GDH antigen testing therefore offers a better method for rul-

Table 3. Performance characteristics of different C. difficile testing algorithms compared to a combined standard. ${ }^{a}$

\begin{tabular}{|c|c|c|c|c|}
\hline \multirow[b]{2}{*}{ Assayb $^{b}$} & \multicolumn{4}{|c|}{ Performance parameter, $\%$ ( $95 \%$ confidence interval) } \\
\hline & Sensitivity & Specificity & PPV ${ }^{c}$ & NPV $^{d}$ \\
\hline GDH, Toxin EIA & $82.9(65.7-92.8)$ & $100(98.5-100)$ & $100(85.4-100)$ & $98.2(95.9-99.3)$ \\
\hline GDH, Toxin EIA, CT & $100(87.9-100)$ & $100(98.5-100)$ & $100(87.7-100)$ & $100(98.5-100)$ \\
\hline GDH, Toxin EIA, PCR & $91.4(75.8-97.8)$ & $97.5(95.0-98.8)$ & $80(63.9-90.4)$ & $99.1(97.0-99.8)$ \\
\hline $\mathrm{GDH}, \mathrm{CT}$ & $100(87.9-100)$ & $100(98.5-100)$ & $100(87.7-100)$ & $100(98.5-100)$ \\
\hline GDH, PCR & $88.6(72.3-96.3)$ & $97.5(95.0-98.8)$ & $79.5(63.1-90.1)$ & $98.7(96.6-99.6)$ \\
\hline
\end{tabular}

aSee Methods for complete description of standard; ${ }^{\mathrm{G}} \mathrm{GDH}$ : glutamate dehydrogenase antigen assay; ${ }^{\mathrm{c}}$ positive predictive value; ${ }^{\mathrm{d}}$ negative predictive value. Toxin EIA, C. difficile toxin A/B enzyme immunoassay; CT, cell-culture cytotoxicity; PCR, toxin B gene detection by PCR.

ing out CDI in the majority of patients who do not have the disease. Surprisingly, GDH displayed better specificity and positive predictive value in our evaluation (93.2\% and 61.4\%, respectively) than the toxin EIA even though this method detects both toxin-producing and non-toxin-producing strains of $C$. difficile. This method offers some of the same advantages as toxin EIA methods including low cost, limited required technical expertise, and rapid turn-around time. However, because this method detects both toxin-producing and nontoxin-producing strains of $C$. difficile, a followup assay to confirm the presence/production of toxin is required for all positive results.

Cell culture cytotoxicity neutralization demonstrates excellent sensitivity and specificity and has often been used as the gold standard for detection of toxin-producing $C$. difficile. ${ }^{7,11,12,14-20}$ However, this assay is also labor intensive, expensive, and slow, requiring up to three days for a result. Although media with preservatives like Cary-Blair are not recommended for the performance of CT, the sensitivity and specificity of CT in this study were each $100 \%$. However, these results should be interpreted carefully because the accuracy of CT was favored in the interpretation of discrepant results based on the superior performance of this assay in previous studies.

Real-time PCR-amplification of the toxin B gene is the most recent method employed for the detection of toxin-producing $C$. difficile. While somewhat expensive, PCR shows the potential to be a rapid and specific means of diagnosing CDI that is amenable to automation. Three small studies using in-house developed RT-PCRs have demonstrated sensitivities of $87.1-91.5 \%,{ }^{14,15,18}$ while studies using the BD Gene0hm PCR assay have shown sensitivities of $83.6-96.4 \%{ }^{8,13,21} \mathrm{~A}$ recent large study reported a sensitivity of $94.4 \%$ using another commercial assay (Xpert $C$. difficile PCR assay; Cepheid, Sunnyvale, CA, USA).${ }^{19}$ This assay was also superior to either EIA alone or an algorithm consisting of GDH and toxin EIA (with CT for all negative EIA results).

Our experience with the application of the BD GeneOhm real-time PCR assay to specimens collected in Cary-Blair medium and frozen was more disappointing with a sensitivity and specificity of $74.3 \%$ and $96.6 \%$, respectively. The poor sensitivity of PCR may relate to freeze-thawing of the original specimen as the PCR assay performance appeared to be optimized when used with fresh stool samples in Cary-Blair Transport. Previous studies have successfully employed PCR on frozen samples, including frozen stool specimens. ${ }^{22}$ While an adverse impact on the accuracy of latex agglutination for the detection of $C$. difficile has been reported, ${ }^{23}$ in at least one study, freezing of stool specimens did not appear to adversely impact the accuracy of either a dot immunobinding or latex agglutination. ${ }^{24}$ In addition, unlike latex agglutination, PCR testing is based on the presence of nucleic acid not antigen which may be affected differently by freezing. However, in this study, the sensitivty of PCR improved to $100 \%$ when performed on fresh samples compared to $74.3 \%$ on frozen samples. Dilution of the fresh sample when placed in the transport medium did not appear to adversely impact the PCR assay (data not shown). The performance of PCR would also improve if the eight $\mathrm{GDH}^{+} / \mathrm{PCR}^{+}$results were considered true positives. Although we chose to classify these samples as false positives for purposes of this evaluation, our experience since that time and the experience of others cited here, ${ }^{8,11,12,20}$ indicate that these can be considered true positives from a clinical perspective. An algorithmic approach may enable laboratories to minimize costs and turnaround time while preserving accuracy by screening samples with a rapid, low-cost modality, and confirming positive results with a more accurate (and more expensive) assay. A two-step algorithm has been successfully employed to rapidly rule out $\mathrm{CDI}$ with negative $\mathrm{GDH}$ results and accurately rule in CDI by performing the cell culture cytotoxicity neutralization assay on all GDH-positive results. ${ }^{7,8}$ Similar algorithms using GDH followed by toxin EIA of positive results have demonstrated excellent specificity, but relatively poor sensitivity. ${ }^{6,12}$ A previous study examined the use of four assays: a GDH antigen specific EIA (C.Diff Chek-60; Techlab, Blacksburg, VA, USA); a lateral flow assay for toxins A and B (C.Diff Quik Chek); a lateral 
flow assay which tests for both GDH and toxins A and B (C.Diff Quik Chek Complete; Techlab, Blacksburg, VA, USA); and a random-access PCR assay for the toxin B gene (Xpert $C$. difficile PCR assay; Cepheid, Sunnyvale, CA, USA).$^{20}$ The C.Diff Quik Chek Complete assay demonstrated a sensitivity of $60 \%$ using a gold standard based on positivity for GDH, toxin EIA, and PCR - each by at least one method. The Xpert PCR assay was used to successfully resolve discrepant results, demonstrating a sensitivity of $100 \%$ and a specificity of $99.6 \%$.

Although a formal cost and time analysis was beyond the scope of this evaluation, the apparent savings in cost and time achieved through an algorithmic approach were evident. A retrospective analysis of different algorithms using the data generated in this evaluation is depicted in Table 3. GDH represents an excellent initial step as a result of its $100 \%$ sensitivity, low cost, fast turn-around time, and simple execution. In our experience, CDI can be quickly and inexpensively ruled out in $84 \%$ of patients. Our results showed that GDH followed by CT of all positive results displays $100 \%$ sensitivity and specificity. While other algorithms offer somewhat inferior sensitivity and specificity, the improved turn-around time, reduced technologist time, and potential cost containment of these approaches may make them more attractive to some clinical laboratories. A two-step algorithm with GDH followed by PCR (after freezing) results in a sensitivity and specificity of $88.6 \%$ and $97.5 \%$, respectively. As the freezing of samples appears to have a negative impact on the accuracy of PCR, an algorithm employing GDH followed by PCR on fresh (non-frozen) specimens is likely to perform much better.

Three-step algorithms involve initial testing of all specimens with the GDH assay followed by toxin EIA of all GDH positive samples. Based on the poor sensitivity of toxin EIA, specimens that are GDH positive and toxin EIA negative should then be tested by a third method. An algorithm using CT as the third method resulted in $100 \%$ sensitivity and specificity. The use of PCR as the third method yielded an improvement in sensitivity relative to the GDH/Toxin EIA two-step algorithm (91.4\% vs $82.9 \%)$, but had a lower specificity (97.5\% vs $100 \%)$. However, the accuracy of this three-step approach is likely to improve if PCR is performed on fresh specimens. Both three-step algorithms would require three tests on 40 of the 57 specimens that are GDH positive. Based on these results, a three-step algorithm does not appear to offer a significant advantage over a two-step approach. However, algorithms utilizing GDH/toxin EIA combination tests (such as C DIFF QUIK CHECK Complete) for initial screening, followed by CT or PCR for confirmatory testing, may provide an alternative approach that utilizes three tests but are per- formed in only two steps.

The major weakness of this study and most studies pertaining to $C$. difficile testing is the lack of a suitable gold standard. Some studies have simply assigned CT as the gold standard, ${ }^{7,11,12,14-20}$ while some have utilized culture of $C$. difficile followed by toxin testing. ${ }^{6,8,12,13,21}$ The latter method is very sensitive and specific but is also slow, expensive, and labor intensive. Other studies advocate assessing a positive final result based on the combination of laboratory findings. While this approach incorporates more information, it is vulnerable to biases concerning the accuracy of the methods under evaluation.

The American Society for Microbiology (ASM) has recently published guidelines for $C$. difficile testing. ${ }^{25}$ Based on these guidelines samples may be screened with a GDH EIA. Samples that then test positive by either a toxin A/B EIA, CT, or a nucleic acid amplification test such as PCR can then be reported positive. Nucleic acid amplification tests may also be performed as a stand-alone assay. Although it is not always feasible to perform in a clinical setting, toxigenic culture is considered the current gold standards based on these criteria.

Previous studies have demonstrated substantial variation in $C$. difficile toxin test performance characteristics. These discrepancies likely result from different commercial tests within particular testing modality, variation in test performance and personnel, and differences in patient populations. Because this is a single-center study performed over a twomonth period in 2009 , it is vulnerable to similar limitations. What is more intriguing, however, is the possibility that geographic differences in $C$. difficile isolates may impact the performance of the same tests used in different laboratories. This hypothesis requires further investigation, and is being actively pursued in our laboratory.

The results of this study confirm that the most commonly used method - the toxin EIA has a relatively poor sensitivity and specificity. The sensitivity of PCR was relatively poor in the setting of specimens collected in CaryBlair transport medium and temporarily frozen. However, PCR results obtained from fresh stool collected in this transport medium appeared much more promising. Based on a retrospective analysis of different algorithms using the data generated in this evaluation, a three-step algorithm does not appear to offer a significant advantage over a two-step approach. Overall, the most practical and balanced approach among the algorithms investigated in this study appears to be a two-step algorithm with initial GDH testing of all specimens followed by either cell culture cytotoxicity neutralization or PCR performed on unfrozen samples. While PCR on all stool specimens currently carries a high cost/test, this method may offer a more rapid alternative to CT for the confirmation GDH-positive samples.

\section{References}

1. Ricciardi R, Rothenberger DA, Madoff RD, Baxter NN. Increasing prevalence and severity of Clostridium difficile colitis in hospitalized patients in the United States. Arch Surg 2007;142:624-31.

2. Kyne L, Hamel MB, Polavaram R, Kelly CP. Health care costs and mortality associated with nosocomial diarrhea due to Clostridium difficile. Clin Infect Dis 2002; 34:346-53.

3. Wilcox MH, Cunniffe JG, Trundle C, Redpath C. Financial burden of hospitalacquired Clostridium difficile infection. J Hosp Infect 1996;34:23-30.

4. Gülke I, Pfeifer G, Liese J, et al. Characterization of the enzymatic component of the ADP-ribosyltransferase toxin CDTa from Clostridium difficile. Infect Immun 2001;69:6004-11.

5. Brazier JS. The diagnosis of Clostridium difficile-associated disease. J Antimicrob Chemother 1998;4:29-40.

6. Fenner L, Widmer AF, Goy G, et al. Rapid and reliable diagnostic algorithm for detection of Clostridium difficile. J Clin Microbiol 2007;46:328-30.

7. Gilligan PH. Is a two-step glutamate dehydrogenase antigen-cytotoxicity neutralization assay algorithm superior to the Premier toxin A and B enzyme immunoassay for laboratory detection of Clostridium difficile? J Clin Microbiol 2008;46:1523-5.

8. Kvach EJ, Ferguson D, Riska PF, Landry ML. Comparison of BD GeneOhm Cdiff real-time PCR assay with a two-step algorithm and a toxin A/B enzyme-linked immunosorbent assay for diagnosis of toxigenic Clostridium difficile infection. J Clin Microbiol 2010;48:109-14.

9. Lipson SM, Tortora G, Tempone A, et al. Rapid Detection of Clostridium difficile in stool using the VIDAS C. difficile toxin A II assay. Diagn. Microbiol. Infect Dis 2003;45:117-21.

10. Mylonakis E, Ryan ET, Calderwood SB. Clostridium difficile-associated diarrhea: a review. Arch Intern Med 2001;161:52533.

11. Novak-Weekley SM, Hollingsworth MH. Comparison of premier toxin A and B assay and the TOX A/B II assay for the diagnosis of Clostridium difficile infection. Clin Vaccine Immunol 2008;15:575-8.

12. Snell H, Ramos M, Longo S, et al. Performance of the TechLab C. DIFF CHEK-60 enzyme immunoassay (EIA) in combination with the $\mathrm{C}$. difficile Tox A/B II 
EIA kit, the Triage C. difficile panel immunoassay, and a cytotoxin assay for diagnosis of Clostridium difficile-associated diarrhea. J Clin Microbiol 2004;42:48635.

13. Stamper PD, Alcabasa R, Aird D, et al. Comparison of a commercial real-time PCR assay for tcdB detection to a cell culture cytotoxicity assay and toxigenic culture for direct detection of toxin-producing Clostridium difficile in clinical samples. J Clin Microbiol 2009;47:373-8.

14. van den Berg RJ, van Coppenraet L, Gerristen H, et al. Prospective multicenter evaluation of a new immunoassay and real-time PCR for rapid diagnosis of Clostridium difficile-associated diarrhea in hospitalized patients. $\mathrm{J}$ Clin Microbiol 2005;43:5338-40.

15. van den Berg RJ, Vaessen N, Endtz HP, et al. Evaluation of real-time PCR and conventional diagnostic methods for the detection of Clostridium difficile-associated diarrhoea in a prospective multicentre study. J Med Microbiol 2007;56:36-42.

16. Reller ME, Lema CA, Perl TM, et al. Yield of stool culture with isolate toxin testing versus a two-step algorithm including toxin testing for detection of toxigenic
Clostridium difficile. J Clin Microbiol 2007;45:3601-5.

17. Ticehurst JR, Aird DZ, Darn LM, et al. Effective detection of toxigenic Clostridium difficile by a two-step algorithm including tests for antigen and cytotoxin. $\mathrm{J}$ Clin Microbiol 2006;44:1145-9.

18. Guilbault C, Labbé AC, Poirier L, et al. Development and evaluation of a PCR method for detection of the Clostridium difficile toxin B gene in stool specimens. J Clin Microbiol 2002;40:2288-90.

19. Novak-Weekley SM, Marlowe EM, Miller $\mathrm{JM}$, et al. Clostridium difficile testing in the clinical laboratory by use of multiple testing algorithms. J Clin Microbiol 2010; 48:889-93.

20. Sharp SE, Ruden LO, Pohl JC, et al. Evaluation of the C. Diff Quik Check Complete assay, a new glutamate dehydrogenase and $\mathrm{A} / \mathrm{B}$ toxin combination lateral flow assay for use in rapid, simple diagnosis of Clostridium difficile disease. J Clin Microbiol 2010;48:2082-6.

21. Terhes G, Urbán E, Sóki J, et al. Comparison of a rapid molecular method, the BD GeneOhm Cdiff assay, to the most frequently used laboratory tests for detection of toxin-producing Clostridium diffi- cile in diarrheal feces. $\mathbf{J}$ Clin Microbiol 2009;47:3478-81.

22. Nechvatal JM, Ram JL, Basson MD, et al. Fecal collection, ambient preservation, and DNA extraction for PCR amplification of bacterial and human markers from human feces. J Microbiol Methods 2008: 72:124-32.

23. Peterson LR, Holter JJ, Shanholtzer CJ, et al. Detection of Clostridium difficile toxins A (enterotoxin) and B (cytotoxin) in clinical specimens. Evaluation of a latex agglutination test. Am J Clin Pathol 1986;86: 208-11.

24. Woods GL, Iwen PC. Comparison of a dot immunobinding assay, latex agglutination, and cytotoxin assay for laboratory diagnosis of Clostridium difficile-associated diarrhea. J Clin Microbiol 1999;28:855-7.

25. Sharp S, Gilligan P. A practical guidance document for the laboratory detection of toxigenic Clostridium difficile [Internet]. Washington, D.C.: PSAB Committee on Laboratory Practices, American Society for Microbiology; 2010 [cited 2011, Jan 8]. Available from: http://www.asm.org/ index.php/policy/public-health.html? title $=$ Public + Health 